\title{
On the distribution of relative humidity in cirrus clouds
}

\author{
P. Spichtinger ${ }^{1}$, K. Gierens ${ }^{1}$, H. G. J. Smit ${ }^{2}$, J. Ovarlez ${ }^{3}$, and J.-F. Gayet ${ }^{4}$ \\ ${ }^{1}$ Deutsches Zentrum für Luft- und Raumfahrt, Institut für Physik der Atmosphäre, Oberpfaffenhofen, Germany \\ ${ }^{2}$ Forschungszentrum Jülich, Institut für Chemie und Dynamik der Geosphäre (ICG-II: Troposphäre), Jülich, Germany \\ ${ }^{3}$ Laboratoire de Météorologie Dynamique du CNRS, Ecole Polytechnique, Palaiseau, France \\ ${ }^{4}$ Laboratoire de Météorologie Physique, Université Blaise Pascal, Clermont-Ferrand, France
}

Received: 15 December 2003 - Published in Atmos. Chem. Phys. Discuss.: 11 January 2004

Revised: 1 April 2004 - Accepted: 1 April 2004 - Published: 16 April 2004

\begin{abstract}
We have analysed relative humidity statistics from measurements in cirrus clouds taken unintentionally during the Measurement of OZone by Airbus In-service airCraft project (MOZAIC). The shapes of the in-cloud humidity distributions change from nearly symmetric in relatively warm cirrus (warmer than $-40^{\circ} \mathrm{C}$ ) to considerably positively skew (i.e. towards high humidities) in colder clouds. These results are in agreement to findings obtained recently from the INterhemispheric differences in Cirrus properties from Anthropogenic emissions (INCA) campaign (Ovarlez et al., 2002). We interprete the temperature dependence of the shapes of the humidity distributions as an effect of the length of time a cirrus cloud needs from formation to a mature equilibrium stage, where the humidity is close to saturation. The duration of this transitional period increases with decreasing temperature. Hence cold cirrus clouds are more often met in the transitional stage than warm clouds.
\end{abstract}

\section{Introduction}

The formation of cirrus clouds in the upper troposphere requires that the relative humidity (with respect to ice, $\mathrm{RHi}$ ) exceeds certain freezing thresholds. These are generally much higher than 100\%; for instance, homogeneous freezing of aqueous solution droplets at temperatures below the supercooling limit of pure water $\left(\approx-40^{\circ} \mathrm{C}\right)$ needs $\mathrm{RHi}>140 \%$ (Koop et al., 2000). Cirrus formation and its subsequent evolution into a mature cirrus cloud (where $\mathrm{RHi}$ is close to saturation) affects the ambient relative humidity field, and it is possible to conclude on cirrus formation pathways by investigation of their ambient $R H i$-distribution (Haag et al., 2003). It is obvious that the humidity within a cloud is even stronger affected by the cloud since it is directly involved in the mi-

Correspondence to: P. Spichtinger

(peter.spichtinger@dlr.de) crophysical processes. It is then clear that the microphysical processes within a cloud shape the statistical distribution of the $R H i$-field. Therefore it should principally be possible to gain insight into the microphysical processes by considering the $R H i$-statistics within clouds.

The statistical distribution of the relative humidity with respect to ice within cirrus clouds was investigated by Ovarlez et al. (2002) using data obtained during the INCA campaigns in the southern (Punta Arenas, Chile, $55^{\circ} \mathrm{S}$ ) and northern (Prestwick, Scotland, $55^{\circ} \mathrm{N}$ ) hemispheres, respectively. The distinction between in-cloud and out-of-cloud situations was made on the basis of the extinction coefficient measured with a polar nephelometer (Gayet et al., 1997): An extinction coefficient of less than $0.05 \mathrm{~km}^{-1}$ was considered a cloud free situation. This corresponds roughly to an ice crystal concentration of $50-100$ particles $\mathrm{L}^{-1}$ of $5 \mu \mathrm{m}$ diameter. Ovarlez et al. (2002) found essentially that two types of distributions can be well fitted to the observations. These are a Gaussian distribution for cirrus warmer than $-40^{\circ} \mathrm{C}$ and a Rayleigh distribution for cirrus colder than $-40^{\circ} \mathrm{C}$. The main point to note here is rather the symmetry of the respective distribution than the type of the distribution itself (which should be considered merely a convenient mathematical expression for the fits). Warmer clouds possess symmetric or quasi-symmetric distributions of $R H i$ centred about $100 \%$ (exemplified by the Gaussian) whereas colder clouds possess distributions of $R H i$ with positive skewness (exemplified by the Rayleigh distribution), i.e. they have a tail towards higher values. This tail might be interpreted a signature of clouds in statu nascendi, where the supersaturation has not yet relaxed to a value close to equilibrium (i.e. saturation). Ovarlez et al. (2002) found slight differences between the in-cloud humidity distributions obtained at the two locations, with a tendency for higher values of $\mathrm{RHi}$ in the southern hemisphere.

In the present paper we analyse humidity data from another data source, namely from the Measurement of OZone by Airbus In-service airCraft project (MOZAIC, Marenco 


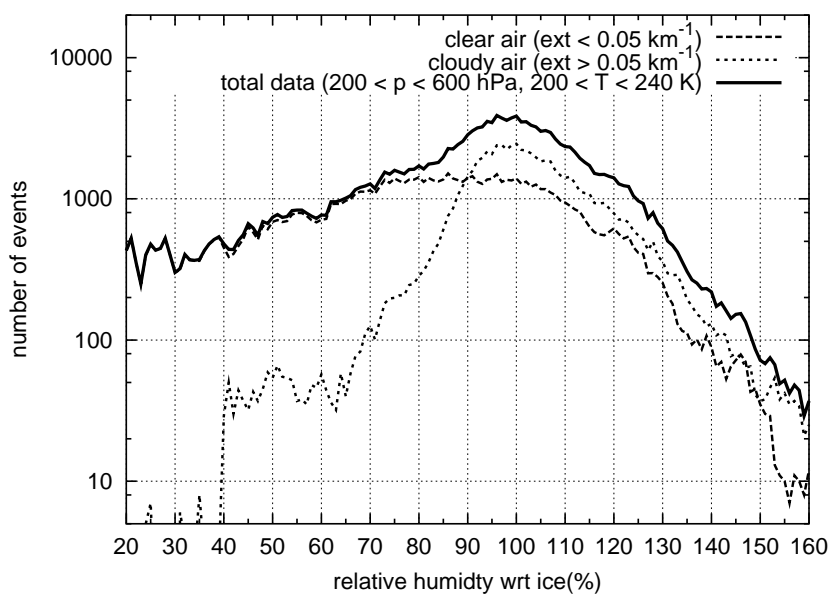

Fig. 1. Statistical distributions (non-normalised) of relative humidity wrt ice inside (dashed line) and outside (dotted line) clouds, and the sum of both (solid line), obtained from INCA measurements. Obviously the bulge in the "sum" distribution originates from measurements inside clouds. It should also be noted that the slopes of the distributions at humidities above ice saturation are similar.

et al., 1998; Helten et al., 1998) and we will show that these data are consistent with the results of Ovarlez et al. (2002).

\section{Data handling}

For the present investigation we use the statistical data of relative humidity with respect to ice in the (mostly northern hemispheric) tropopause region as obtained from MOZAIC aircraft (Gierens et al., 1999). For this data set it is not really possible to decide whether a recording that signals supersaturation comes from cloud free air or from within a cirrus cloud. Thus the humidity statistics obtained from the data set bears signatures from both cloudy and clear air. Of course, data from substantially subsaturated air are obviously obtained in clear regions. The common characteristics of all humidity statistics obtained from these data sets is a relatively flat exponential distribution for the subsaturated air (i.e. $20 \% \lesssim R H i \lesssim 80 \%$ ) and a steeper exponential distribution in supersaturated air masses. These characteristics can also be found in humidity statistics obtained from the microwave limb sounder (MLS) on board the Upper Atmosphere Research Satellite (UARS), where a cloud clearing could be performed successfully (Spichtinger et al., 2002, 2003). Hence, the exponential parts of the humidity statistics are characteristic for cloud free air. The signature of clouds in the MOZAIC data is a "bulge" around saturation (i.e. $R H i \approx 100 \pm 20 \%$ ). Such a bulge is not present in the cloud cleared MLS data. Whereas we were interested in the exponential parts of the humidity statistics in our previous papers, we will here consider the "cloud bulge" in more detail.
The interpretation of the bulges as a cloud signature can be underpinned by taking a look at data from the INCA project, namely at the combination of humidity data from the frostpoint hygrometer (Ovarlez et al., 2002) and extinction data from the nephelometer (Gayet et al., 1997). The combination allows to distinguish in-cloud from out-of-cloud data records: As in the work of Ovarlez et al. (2002) we fix the cloud threshold at an extinction of $0.05 \mathrm{~km}^{-1}$. Using all measurements in the pressure range $200 \leq p \leq 600 \mathrm{hPa}$ and in the temperature range $200 \leq T \leq 240 \mathrm{~K}$, we have derived three statistical distributions of relative humidity: inside clouds, outside clouds, and irrespective of cloud presence (i.e. the sum of the two others). These distributions are shown in Fig. 1. The relative humidity distribution of cloud free data shows the usual characteristic of tropospheric data (see e.g. Gierens et al., 1999; Spichtinger et al., 2002). The shape of the distribution can be described using two exponential distributions with different slopes. As we expected, there is a kink at saturation. In contrast the distribution obtained from the cloudy data has the shape as described in Ovarlez et al. (2002): The distribution is centred at saturation and the frequency of occurrence of relative humidity decreases towards lower and higher humidities. The most interesting distribution for our present purpose is that obtained from the sum. This distribution has qualitatively the same shape as the distributions obtained from the MOZAIC data: There is the characteristic shape of the pair of exponential distributions (typically for tropospheric data) but there is also a bulge around saturation. This bulge is the result of the in-cloud data which is evident from the figure.

In order to investigate the cloud bulges in MOZAIC data we treat the data in the following way: First we run a moving average (with a window width of $5 \% \mathrm{RHi}$ ) over the respective distribution to reduce their statistical noise. Then we construct baselines representing the exponential parts of each distribution and subtract them from their respective distribution of $\mathrm{RHi}$. The residuum from this operation is the bulge alone. This baseline is constructed in the following way: On the left and the right of the bulge there are the exponentials with their different slopes ( 2 free parameters). These two exponentials are then smoothly connected by means of an "exponential" with varying exponent. The varying exponent is a Fermi function centred at a value close to $100 \%$ (1 free parameter). The width of the Fermi function is adjustable (1 free parameter). The whole baseline function is scaled with another adjustable parameter, such that there are a total of 5 free parameters. The functional form of the baseline is then:

$$
B(x)=N \exp \left[-F(x) \cdot\left(x-x_{c}\right)\right]
$$

with the Fermi function

$F(x)=a+\frac{b-a}{1+e^{-c\left(x-x_{c}\right)}}$.

Obviously, the limiting values for large negative (subsaturation) and large positive values (supersaturation) of $x-x_{c}$ 


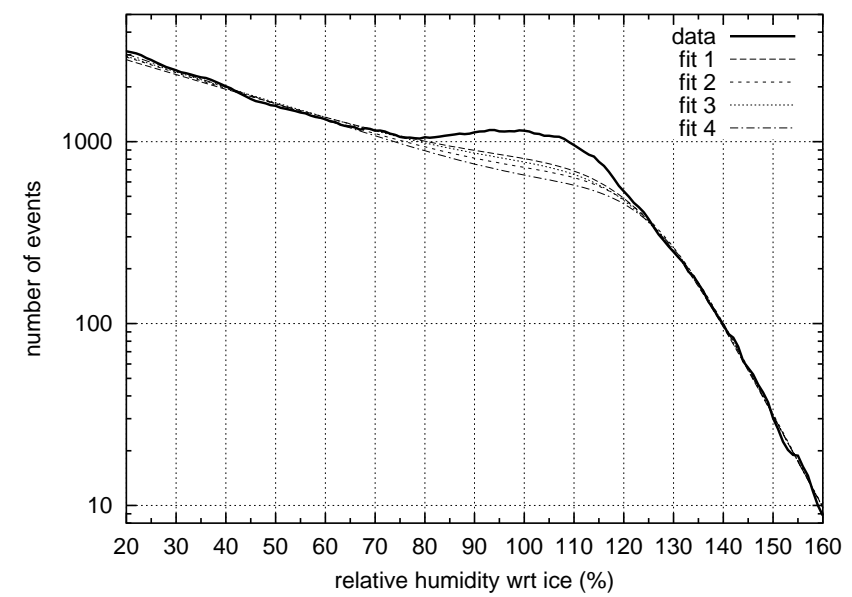

Fig. 2. Examples of some baseline fits.

are $a$ and $b$, respectively, which are the slopes of the corresponding exponential distributions. $x_{c}$ is the value where the Fermi function is centred, and $c$ determines the sharpness of the transition between the two slopes $a$ and $b . N$ is the scale parameter. These five free parameters $\left(\left\{a, b, c, x_{c}, N\right\}\right)$ are determined numerically using a simple optimisation routine, that aims at minimising the sum of squared differences between the baseline fit and the data in the two $\mathrm{RHi}$ regions where the distribution is exponential.

After subtraction of the baseline the cloud bulge plus some residual noise remains and can be studied further. This is done in the next section. Certainly, the remaining bulge is sensitive to the construction of the baseline and to the parameters. For studying the impact of baseline construction on the bulge we have used the following procedure: For each distribution of $R H i$ we have constructed several baselines distinguished by different ranges of best fit in the exponential parts, e.g. $30-70 \%$ or $30-80 \%$ etc. The standard range for the calculations was $40-80 \% \mathrm{RHi}$ and $120-160 \% \mathrm{RHi}$. Within these ranges the distributions obviously follow exponential distributions. The different fit ranges per se imply differences in the goodness-of-fit measure $\chi^{2}$. Therefore we use for comparison of the quality of the fits a normalised $\chi_{R H i}^{2}:=100 \% \cdot \frac{\chi^{2}}{\Delta R H i}$ were $\Delta R H i$ denotes the range within the baseline was constructed. With this variable we are able to determine the best baseline and using the distinct baselines we can study the variations of baseline construction and their impact on the bulge. An example of some different baseline fittings is given in Fig. 2. The corresponding bulges after subtraction of these various baselines are shown in Fig. 3.

We interprete the bulges or the difference distributions as cloud signatures and as distribution of relative humidity inside clouds. But, as one can see in the figures, the residual number of events after baseline subtraction are sometimes negative, which simply is a consequence of the fact that it is not strictly possible to discern cloudy from non-cloudy data

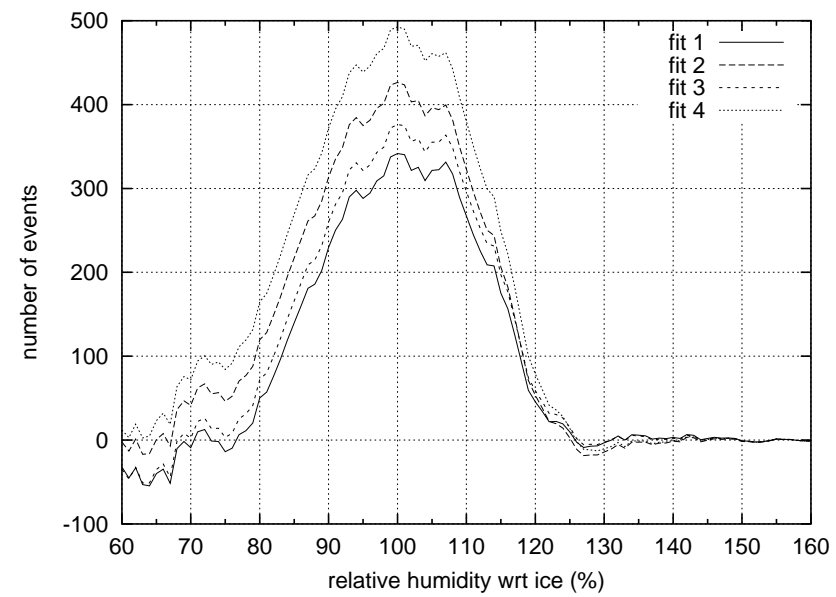

Fig. 3. Examples of the remaining bulges (or the difference distributions) after subtracting the baseline fits in Fig. 2.

in MOZAIC, that is, the baselines are too close to the data. This indicates, that with the baselines subtracted we probably also remove in-cloud data, especially in the supersaturated region. As (Fig. 1) shows, the slopes of the humidity distributions above ice saturation in the in-cloud and out-of-cloud INCA data are similar, which could mean that by the baseline subtraction we remove from all supersaturation bins nearly a constant (but here unknown) fraction of in-cloud data. However, since there is no possibility to flag cloudy data, we do not see a better possibility of baseline construction. Thus we have to accept that we miss some of the cloudy data and that we also have negative values in the residuals, which we will set to zero for the further analysis.

For analysis of the difference distributions we calculate the mean values, standard deviations and the so-called Lskewness (for a definition see the appendix). We use the Lskewness instead of the usual skewness because of its greater robustness against outliers (see e.g. Guttman, 1993), which is necessary here because there is still some noise in the bulge data even after the initial smoothing. The traditional skewness is very sensitive to such noise and can therefore not be used as a reliable measure.

These statistical measures are calculated in the range 70 $150 \% R H i$, which per se introduces a certain positive skewness even in a perfectly symmetric distribution (see below). The lower boundary is considered a lower threshold where most cirrus clouds will be evaporated completely. The upper boundary is a typical threshold for homogeneous ice nucleation in the upper troposphere (see Koop et al., 2000); higher thresholds apply for still colder temperatures, but the data get more noisy, hence we constrain the range for our calculations to $150 \%$ and do not go beyond. Since we constrain the calculation of mean, standard deviation and L-skewness to this range which is asymmetric with respect to saturation, we have to determine how this affects in particular the calculation of the skewness. In order to estimate this effect we 

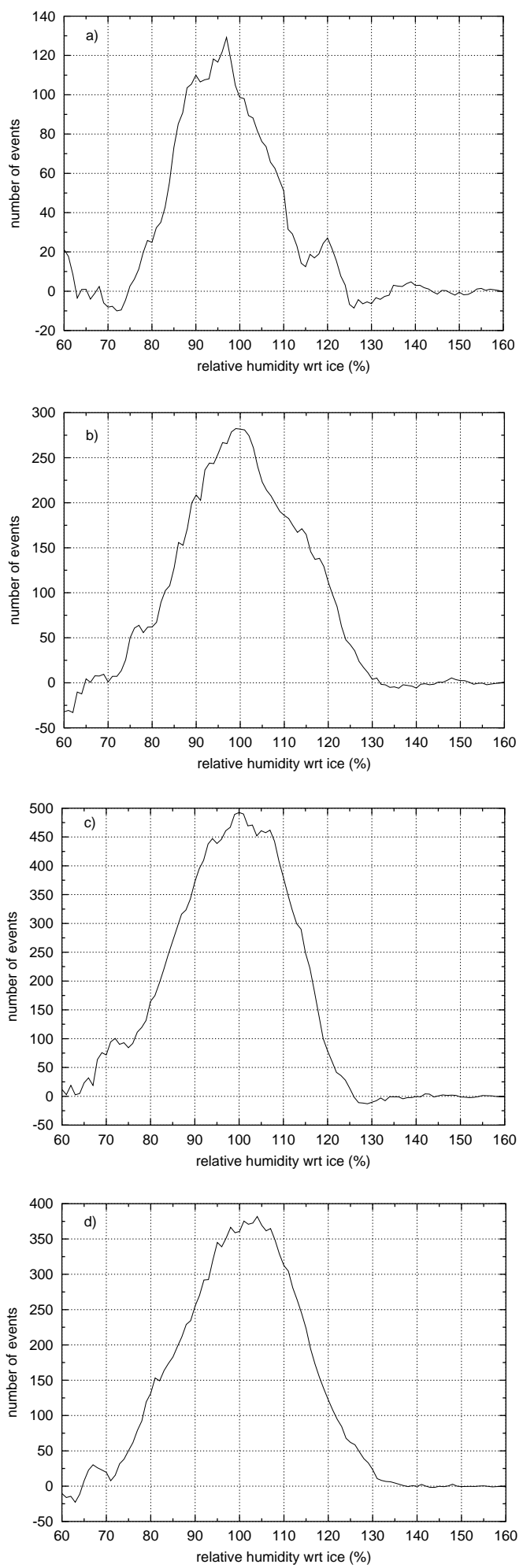

Fig. 4. Non-normalised probability distribution of relative humidity over ice in tropospheric tropical (south of $30^{\circ} \mathrm{N}$ ) MOZAIC data, after baseline subtraction for 4 pressure levels: (a) 190-209, (b) 210-230, (c) 231-245, (d) 246-270 hPa. analyse a Gaussian (i.e. symmetric) distribution in the range 70-150\% RHi with and without an additional perturbation at $150 \% \mathrm{RHi}$ (about $5 \%$ of the maximum). Perturbations of the order $5 \%$ of the maximum at $150 \% R \mathrm{Ri}$ is what we normally have in our data after smoothing. This Gaussian distribution

$f_{X}(x)=\frac{1}{\sqrt{2 \pi \sigma_{0}}} \exp \left(-\frac{1}{2}\left(\frac{x-\mu_{0}}{\sigma_{0}}\right)^{2}\right)$

is centred at $\mu_{0}=100 \% R H i$ and the parameter $\sigma_{0}=11.25 \%$ $R H i$ is chosen such that the standard deviation (for the range $70-150 \% R \mathrm{Hi}$ ) is similar to those determined for the bulges. If we now compute the statistical measures in the restricted range $70-150 \% R H i$, we find the mean value in the range $100.11 \leq \mu \leq 100.19 \% R H i$ (the greater value arises when the noise peak at $150 \%$ is added), and the standard deviation in the range $11.10 \leq \sigma \leq 11.28 \% \mathrm{RH}$. The most important result is that the L-skewness $\tau_{3}$ ranges within: $0.0077 \leq \tau_{3} \leq 0.0161$ (for a symmetric distribution the L-skewness is zero by definition). Hence, in the discussion of the results a distribution with $\tau_{3} \leq 0.0161$ can be classified as nearly symmetric, a distribution with $\tau_{3}>0.0161$ can be classified as asymmetric.

\section{Results}

\subsection{MOZAIC data}

Let us first consider MOZAIC data recorded south of $30^{\circ} \mathrm{N}$ (tropical data) between 1995 and 1999. We show tropospheric data from four pressure levels 190-209, 210 230, 231-245, and 246-270 hPa (hereafter levels 1-4). All these are characterised by a rather narrow temperature distribution. The mean temperatures on the four levels are $-54,-49,-44,-39^{\circ} \mathrm{C}$, respectively, the standard deviations range between $2.0^{\circ} \mathrm{C}$ and $3.3^{\circ} \mathrm{C}$. Hence, using these levels there is a splitting of the data in distinct temperature classes. After applying the procedure described in Sect. 2 we see that for all different baseline fits the structure of the difference distributions remains mainly the same. Hence, it is acceptable to consider the best fit for describing the structure of the distributions. For evaluating the distributions more quantitatively we consider also the variations of the baseline fits, particularly the varying L-skewness. The measured humidity distributions of the cloud bulges (after baseline subtraction of the best fit, i.e. minimising $\chi_{R H i}^{2}$ ) are presented in Fig. 4.

It can be seen that after baseline subtraction the residual number of events is rather small compared to the original data base (cf. the numbers along the y-axis of Fig. 2). But nevertheless, the distributions contain a considerable fraction of the total data (13-33\%, depending on the pressure level) and the noise in the distributions is quite small (due to the moving average of the $R H i$-distributions). For 
Table 1. Variations of mean values, standard deviations and Lskewness for the difference distributions of the different MOZAIC data sets for distinct baseline fits as described in Sect. 2.

\begin{tabular}{lccc}
\hline data & $\mu(\% \mathrm{RHi})$ & $\sigma(\% \mathrm{RHi})$ & $\tau_{3}$ \\
\hline tropical & & & \\
lev. 1 & $96.86-99.88$ & $10.18-10.50$ & $0.1068-0.1310$ \\
lev. 2 & $97.56-102.96$ & $10.60-12.14$ & $0.0071-0.0598$ \\
lev. 3 & $98.39-100.95$ & $10.22-11.69$ & $-0.0480-0.0084$ \\
lev. 4 & $100.87-101.48$ & $10.71-12.28$ & $-0.0068-0.0057$ \\
\hline extratr. & & & \\
total & $98.44-101.72$ & $9.23-10.77$ & $0.0955-0.1178$ \\
$K_{1}$ & $103.09-105.64$ & $9.20-10.87$ & $0.0882-0.1225$ \\
$K_{2}$ & $99.48-101.61$ & $8.39-9.28$ & $-0.0186-0.0524$ \\
\hline
\end{tabular}

all distributions we see quite the same shape: The distribution is centred around saturation, the mean values range between 97 and $103 \% R H i$ and the standard deviations are about $11 \% \mathrm{RH} i$ (see Table 1). The difference distributions obtained at the two upper pressure levels (level 1 and 2) are clearly skew (i.e. asymmetric), distributions from the two lower levels (level 3 and 4) are symmetric. This result can be verified by the L-skewness: For the two upper pressure levels the L-skewness $\tau_{3}$ takes values in the following intervals: $0.1068 \leq \tau_{3}$ (level 1$) \leq 0.1310$ and $0.0071 \leq \tau_{3}($ level 2$) \leq 0.0598$. Hence, the difference distributions for the upper two levels are asymmetric according to the L-skewness (see Sect. 2). For the two lower pressure levels the L-skewness $\tau_{3}$ ranges between -0.0480 and 0.0084 , therefore we can assume that the distributions are nearly symmetric according to the L-skewness (see Sect. 2).

We now consider tropospheric MOZAIC data recorded north of $30^{\circ} \mathrm{N}$ (extratropical data) between 1995 and 1999 in the pressure range $175 \leq p \leq 275 \mathrm{hPa}$. For this data set there is not such a sharp temperature stratification due to the pressure levels as in the tropical data. Hence, for studying the distributions in distinct temperature classes we split the data in the following way: One class $K_{1}$ contains all data with temperatures in the interval $-55 \leq T \leq-50^{\circ} \mathrm{C}$, and one class $K_{2}$ with temperatures in the interval $-50 \leq T \leq-45^{\circ} \mathrm{C}$. Additionally, we have collected all data (including data from outside the warm and cold classes) to a third class. A more detailed splitting of the data is not reasonable because of the noise that then appears. In Fig. 5 the difference distributions of the three classes (total, $K_{1}$ and $K_{2}$ data) are presented.

As for the tropical data after baseline subtraction the residual number of events is rather small compared with the original data set. But also in these cases the remaining number of data in the difference distributions are large enough to draw some conclusions although the fraction of the remaining data ranges between 2 and $8 \%$ : The total number of data are much higher for the extratropics than for the tropics. The statistical noise is quite small again.
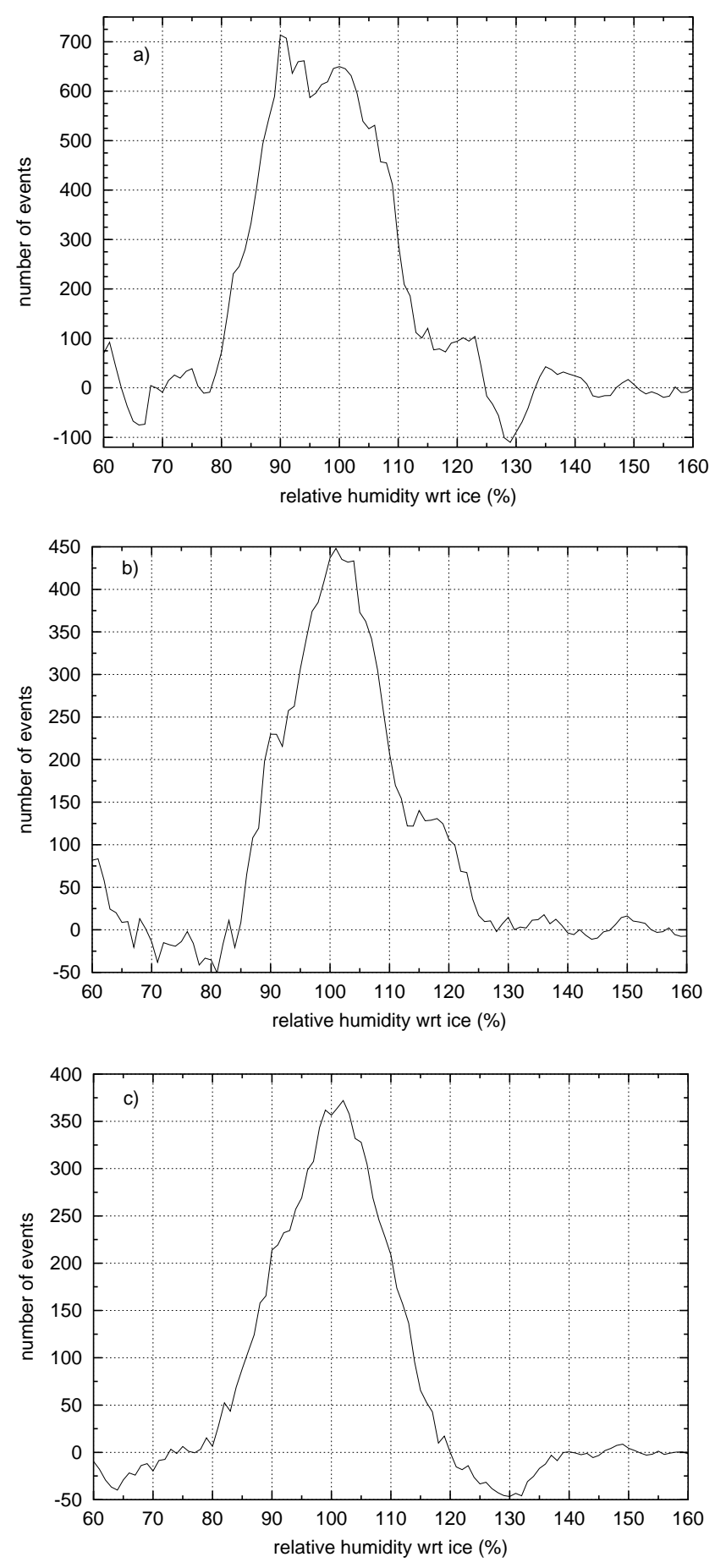

Fig. 5. Non-normalised probability distribution of relative humidity over ice in tropospheric extratropical (north of $30^{\circ} \mathrm{N}$ ) MOZAIC data, after baseline subtraction: (a) total data, (b) class $K_{1}\left(-55 \leq T \leq-50^{\circ} \mathrm{C}\right)$, (c) class $K_{2}\left(-50 \leq T \leq-45^{\circ} \mathrm{C}\right)$.

We can see a similar result as for the tropical distributions: the difference distributions for the three data classes are again centred at saturation, the mean values range between 98 and 


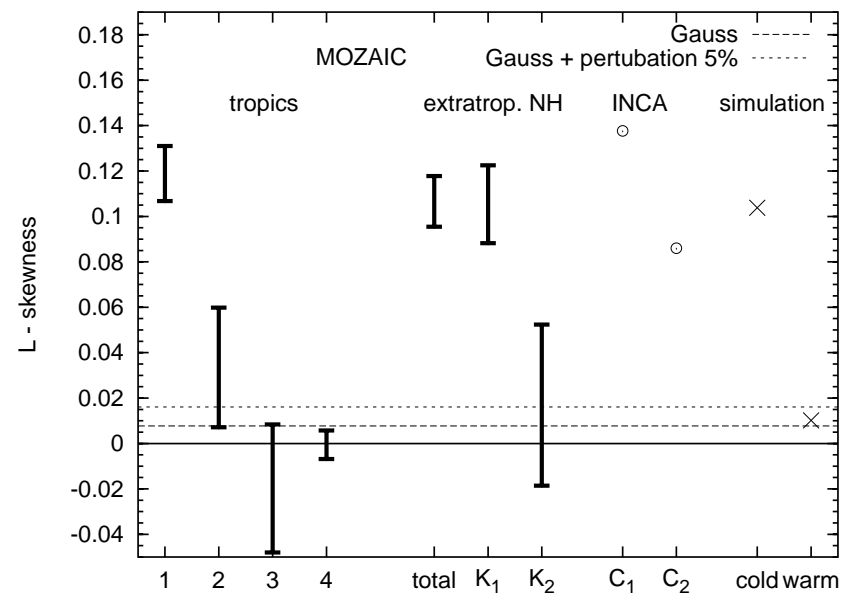

Fig. 6. L-skewness of the difference distributions for the tropospheric MOZAIC data (error bars, see also Sect. 3.1 and Table 1) and the INCA data (circles, see also Sect. 3.2). Additionally, the L-skewness of a Gaussian distribution (without and with a perturbation of $5 \%$ of the maximum at $150 \%$ ) in the range $70-150 \% \mathrm{RHi}$ are shown. These values can be used to distinguish between symmetric and asymmetric distributions. The crosses represent the Lskewnesses resulting from the two numerical experiments of Sect. 4.

$106 \% R \mathrm{Hi}$, the standard deviations range between 8 and $11 \%$ RHi.

The difference distributions obtained from the total extratropic data and the "cold data" are clearly skew, the distribution obtained from the "warm data" is almost symmetric. This is confirmed by the L-skewnesses: For the total data the L-skewness is $0.0955 \leq \tau_{3} \leq 0.1178$, for the "cold data" the Lskewness is $0.0882 \leq \tau_{3} \leq 0.1225$. Hence, these distributions are clearly asymmetric. The L-skewness for the "warm data" is $-0.0186 \leq \tau_{3} \leq 0.0524$ and therefore we can conclude, that this distribution is almost symmetric.

The mean values, standard deviations and L-skewness values are collected in Table 1, the L-skewness values (and their variations) are visualised in Fig. 6 . In this figure additionally the values for a Gaussian distribution and a perturbed Gaussian distribution (see Sect. 2) are shown, hence it is easy to distinguish between the symmetric and asymmetric distributions.

In looking at Fig. 6 one should consider the values displayed as lower estimates, because, as stated before, the range of the computation of the moments was confined at $150 \% R H i$ and cloud events can get lost in our baseline subtraction procedure. Since this happens evidently more probably in the supersaturated than in the subsaturated regime, the underestimation of the true L-skewness is probably the stronger the more asymmetric is the distribution. Thus, we expect that the true contrast between the skewnesses for warm and cold clouds, respectively, is larger than indicated by the error bars in the figure.

\subsection{INCA data}

The INCA campaigns took place in the extratropical latitudes of both hemispheres. Hence, we can compare the distributions of relative humidity in clouds obtained from the INCA data set to the corresponding distributions obtained from the extratropical MOZAIC data. As before we have picked the INCA data out of two different temperature classes: The class $C_{1}$ contains all data in the temperature range $-55 \leq T \leq-50^{\circ} \mathrm{C}$ and the class $C_{2}$ contains all data in the temperature range $-50 \leq T \leq-45^{\circ} \mathrm{C}$. For these two classes we have calculated the L-skewness in the range $70-150 \% R H i$. The values for the two distributions $\left(\tau_{3}\left(C_{1}\right)=0.1377, \tau_{3}\left(C_{2}\right)=0.0860\right)$ are visualised in Fig. 6 . We get the same qualitative effect as for the two difference distributions obtained from the temperature classes $K_{1}$ and $K_{2}$ (MOZAIC): For the colder clouds the distribution is skewer than for the warmer clouds. Comparing these values with the L-skewness obtained from the MOZAIC data (classes $K_{1}$ and $K_{2}$ ) we see that the values of MOZAIC data are a lower approximation due to the causes mentioned in Sect. 2.

\subsection{MLS data}

In order to show as a contrast to the previous data sets an example where cloud clearing works effectively, we show here one example of MLS data analysis for the two nominal pressure levels of $147 \mathrm{hPa}$ and $215 \mathrm{hPa}$ (Spichtinger et al., 2002, 2003). Figure 7 shows that for all concerned tropospheric data sets after baseline subtraction there remains only noise. This can be interpreted that the cloud clearing algorithm described in Spichtinger et al. $(2002,2003)$ works very well and almost no cloudy measurements are left in the data.

\section{Discussion}

Obviously there is a qualitative difference between the incloud distributions of $R H i$ for warm and cold cirrus, respectively. This contrast consists of the different shapes of the distributions, namely symmetric for warm cirrus versus positively skew for cold cirrus. This leads to the question about the physical processes (or possibly selection biases) that produce such qualitatively different distributions of in-cloud relative humidity.

We believe that the difference we see in the humidity distributions is caused by the temperature dependence of the length of time a cirrus cloud needs to approach saturation from an initial high supersaturation at its instant of formation. This transitional period is about twice as long at $-60^{\circ} \mathrm{C}$ than at $-40^{\circ} \mathrm{C}$, because both the diffusivity of water molecules in air and the saturation vapour pressure decrease with decreasing temperature. The nominal crystal growth time scale 
(Kärcher and Solomon, 1999, Eq. B5) in a young cirrus cloud can be written as (Gierens, 2003)

$\tau_{g}=7.14 \times 10^{5} T^{-1.61} p\left[s_{0} e^{*}(T)\right]^{-1 / 3} N^{-2 / 3}$,

with initial supersaturation at cirrus formation $s_{0}$, saturation vapour pressure over ice $e^{*}(T)$ (in $\mathrm{Pa}$ ), and number density of ice crystals formed $N$ (in $\mathrm{m}^{-3}$ ). $\tau_{g}$ is in seconds. Typical growth time scales range from 10 min to half an hour, however, the cirrus transition time to phase equilibrium is more than double that quantity, in particular because initially the condensation rate is very small since the ice crystals are very small. This means that the transition period from cirrus formation to phase equilibrium can make up a substantial fraction of the total cloud life time. In fact, especially thin and sub-visible cirrus in cold air (below about $215 \mathrm{~K}$ ) may not reach equilibrium at all, i.e. the crystals sediment out of the cloud before the in-cloud humidity reaches saturation (Kärcher, 2002). This in turn implies that a substantial fraction of the cirrus clouds probed unintentionally by a MOZAIC aircraft can still be in the transition phase. Since the duration of the transition phase increases with decreasing temperature, the probability to probe a cirrus in the transition phase instead of the equilibrium phase increases with decreasing temperature. From this consideration we would expect, that we find a slightly positively skew (or almost symmetric) distribution of $\mathrm{RHi}$ in warm cirrus, but a strongly skew distribution in cold cirrus. Furthermore, the threshold supersaturation for homogeneous nucleation grows about linearly with decreasing temperature. This additionally leads to a longer relaxation phase for cold than for warm clouds.

Also vertical motions have an influence on the duration of the transition to phase equilibrium. Uplifting motions evidently prolong this period because they reduce the saturation pressure via adiabatic cooling. The effect can be quantified by using the updraft time scale, $\tau_{u}$ (Gierens, 2003):

$\tau_{u}=1.67 \times 10^{-2} w^{-1} T^{2}$,

with vertical velocity $w$. The transition duration increases with decreasing updraft timescale. Hence, strong vertical motion leads to an additional prolongation of the transition period. Unfortunately, the MOZAIC data base contains no information about vertical velocities, therefore this effect cannot be quantified. Additionally, at the same vertical motion the transition duration increases with decreasing temperature, which adds to the microphysical temperature effect mentioned above.

We have performed a simple numerical exercise to simulate the transition process without considering the dynamical effect. The simulation starts with an initial relative humidity of $140 \%$ or $160 \%$, and then the $R H i$ changes in variable steps of about $\pm 1 \%$ according to the sign of a uniformly distributed random number. The range of the random number distribution is slightly asymmetric around zero such that there is always a slightly higher chance that $R H i$ gets closer
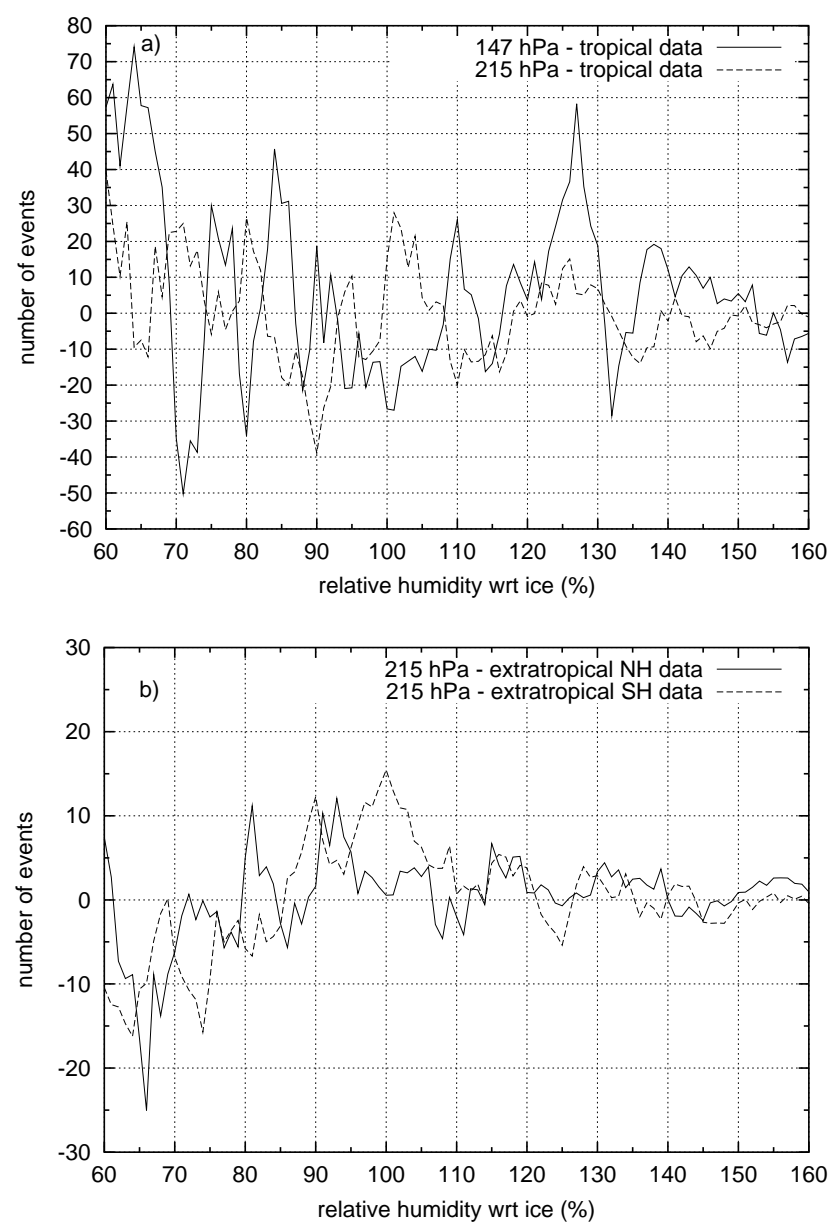

Fig. 7. Non-normalised probability distributions of relative humidity over ice, after baseline subtraction for tropospheric MLS data: (a) tropical (south of $30^{\circ} \mathrm{N}$ ) MLS data on pressure levels 147 (solid) and $215 \mathrm{hPa}$ (dashed) (b) extratropical northern hemispheric (north of $30^{\circ} \mathrm{N}$, solid) and southern hemispheric (south of $30^{\circ} \mathrm{S}$, dashed) MLS data on pressure level $215 \mathrm{hPa}$. Because of cloud clearing there remains after baseline subtraction only a flat distribution of noise along the zero line.

to $100 \%$ than further away. The number of steps is 800 for representation of a cold case, which is a suffiently small number that the system has still a memory of its initial state, that is, it is in a transitional stage. In this case the initial relative humidity was set to $160 \% \mathrm{RHi}$. For the warm case we use 1600 steps (i.e. $2 \times 800$, since one step in the cold case represents about double the time of one step in the warm case, because of the different growth time scales, see above). It turns out that this number of steps is sufficient to loose the memory of the initial state. In this case the initial relative humidity was set to $140 \% R H i$. In order to get smooth statistics, this simulation is repeated 100000 times for each case. We find distributions centred close to $100 \%$ and with standard deviations close to $10 \%$ in both cases (see Fig. 8). Both distributions are positively skew, but the L-skewness in the cold case 


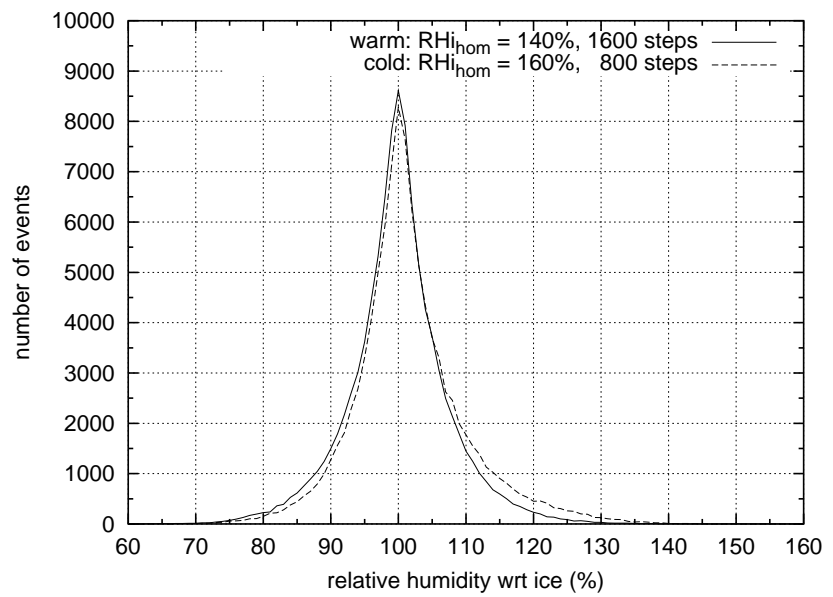

Fig. 8. Simulation of statistical distributions of relative humidity in warm $\left(\mathrm{RHi}_{\text {hom }}=140 \% \mathrm{RHi}, 1600\right.$ steps $)$ and cold ( $R H i_{\text {hom }}=160 \% R H i, 800$ steps) cirrus clouds.

is 10 times larger than in the warm case, since in the cold case there is still a considerable tail in the $R H i$-distribution extending to the initial value of $160 \%$. The distributions in these two cases turn out to have different skewnesses in spite of similar simulated time because the microphysical process rates are different. The L-skewness values of the two simulations are indicated in Fig. 6 as crosses. Hence, the values are similar to the values obtained from the difference distributions of the different data sets. For the skewness of the distributions the main impact is due to the number of steps, i.e. the different growth time. The initial relative humidity only slightly affects the skewness.

Having the INCA data it is the relatively straightforward idea to apply the baseline fitting and subtraction also to this data set and to compare the resulting cloud bulge with the true in-cloud distribution of relative humidity (dotted curve in Fig. 1). Although the amount of INCA data is not sufficient to perform the complete analysis (too much noise!) this test gives interesting results. First, we find that the residual number of events (i.e. the cloud bulge) only represents about $1 / 5$ to $1 / 4$ of the true cloud events found by the nephelometer analysis. Such a fraction might be expected to be characteristic for the MOZAIC data as well. However, the INCA derived fraction cannot be generalised to MOZAIC in a straightforward way because of different measurement strategies, techniques, and hence different selection biases. Second, the out of cloud data in Fig. 1 (dashed curve) can be fitted with a baseline function very well over the total range from 30 to about $150 \%$ RHi (not shown), since it does not display a bulge around saturation. This means that clouds thinner than the nephelometer threshold do not contribute much to a bulge signature, and that the bulge mainly represents thicker clouds. The $R H i$-statistics within thin clouds therefore seems to resemble that of clear air which can result because the relaxation time for thin clouds can be extremely long ( $N$ small in Eq. 3). It can even be longer than the sedimentation time scale for the ice crystals; such clouds do not reach phase equilibrium at all (cf. Kärcher, 2002).

\section{Conclusions}

Statistical distributions of relative humidity with respect to ice in cirrus clouds have been analysed. Humidity data from MOZAIC were taken, baselines were fitted to the ranges where $R H i$ is distributed exponentially, and the residuals after baseline subtraction have been investigated. The residuals, interpreted as data stemming from measurements within cirrus clouds, are unimodal distributions peaked close to saturation, with standard deviations of the order $10 \%$ in relative humidity units. The interpretation of the residuals as cloud signatures is corroborated by corresponding features in the INCA data, where clouds can be detected using nephelometer data.

As in the earlier work of Ovarlez et al. (2002) the shape of the residual distributions (the cloud bulge) turned out to depend on cloud temperature. Whereas we found nearly symmetric distributions in warm cirrus $\left(T>-40^{\circ} \mathrm{C}\right)$, the distributions are clearly positively skew in colder clouds. The skewness seems to increase with decreasing temperature.

Our interpretation of this feature is that warm cirrus clouds probed unintentionally by MOZAIC aircraft are mostly in a mature stage. The signature of this is a symmetric distribution of $R H i$ centred at saturation. On the other hand, cold cirrus probed unintentionally are more often in a transitional state between their instant of formation and their mature stage. The signature of the transitional stage is a tail in the distribution extending from saturation to the threshold relative humidity for freezing. The origin of the difference lies in the different lengths of time a cirrus needs to reach equilibrium via crystal growth after its formation at high supersaturation. The growth time scale decreases with decreasing temperature, such that the time of transition is about twice as long at $-60^{\circ} \mathrm{C}$ than at $-40^{\circ} \mathrm{C}$. This difference is reflected in the different shapes of the humidity distributions within clouds.

\section{Appendix: L-moments}

Formal definition of L-moments:

For this purpose one uses sample probability weighted moments $b_{r}(r=0,1,2,3 \ldots)$. These moments computed from data values $X_{1}, X_{2}, \ldots X_{n}$, arranged in increasing order, are given by

$$
\begin{aligned}
b_{0} & :=\frac{1}{n} \sum_{j=1}^{n} X_{j} \\
b_{r} & :=\frac{1}{n} \sum_{j=r+1}^{n} \frac{(j-1)(j-2) \ldots(j-r)}{(n-1)(n-2) \ldots(n-r)} X_{j}
\end{aligned}
$$


Using these weighted moments $b_{r}$ in combination with the coefficients of the "shifted Legendre polynomials" one can define the so-called L-moments:

$l_{1}:=b_{0}$

$l_{2}:=2 b_{1}-b_{0}$

$l_{3}:=6 b_{2}-6 b_{1}+b_{0}$

By combining these L-moments we can calculate some robust analoga to the usual higher moments in statistics (e.g. skewness or kurtosis). For our purpose only the L-skewness is important:

L-skewness $\quad \tau_{3}:=\frac{l_{3}}{l_{2}}$

For calculating the L-skewness we use the method of Hosking (1990) which is based on order statistics.

Acknowledgements. We thank U. Schumann and B. Kärcher for constructive comments to a draft version of this paper and A. Minikin for providing the INCA data.

\section{References}

Gayet, J.-F., Crépel, O., Fournol, J. F. and Oshchepkov, S.: A new airborne polar Nephelometer for the measurement of optical and microphysical cloud properties, Part I: Theoretical design, Ann. Geophys., 15, 451-459, 1997.

Gierens, K.: On the transition between heterogeneous and homogeneous freezing, Atmos. Chem. Phys., 3, 437-446, 2003.

Gierens, K., Schumann, U., Helten, M., Smit, H. G. J., and Marenco, A.: A distribution law for relative humidity in the upper troposphere and lower stratosphere derived from three years of MOZAIC measurements, Ann. Geophys., 17, 1218-1226, 1999.
Guttman, N. B.: The use of L-moments in the determination of regional precipitation climates, Journal of Climate, 6, 2309-2325, 1993.

Haag, W., Kärcher, B., Ström, J., Minikin, A., Lohmann, U., Ovarlez, J., and Stohl, A.: Freezing thresholds and cirrus cloud formation mechanisms inferred from in situ measurements of relative humidity, Atmos. Chem. Phys., 3, 1791-1806, 2003.

Helten, M., Smit, H. G. J., Sträter, W., Kley, D., Nedelec, P., Zöger, M., and Busen, R.: Calibration and performance of automatic compact instrumentation for the measurement of relative humidity from passenger aircraft, J. Geophys. Res., 103, $25643-$ $25652,1998$.

Hosking, J. R. M.: L-moments: analysis and estimation of distributions using linear combinations of order statistics, J. R. Stat. Soc., Ser. B, 52, 105-124, 1990.

Kärcher, B.: Properties of subvisible cirrus clouds formed by homogeneous freezing, Atmos. Chem. Phys., 2, 161-170, 2002.

Kärcher, B. and Solomon, S.: On the composition and optical extinction of particles in the tropopause region, J. Geophys. Res., 104, 27 441-27 459, 1999.

Koop, T., Luo, B., Tsias, A., and Peter, T.: Water activity as the determinant for homogeneous ice nucleation in aqueous solutions, Nature, 406, 611-614, 2000.

Marenco, A., Thouret, V., Nedelec, P., Smit, H., Helten, M., Kley, D., Karcher, F., Simon, P., Law, K., Pyle, J., Poschmann, G., von Wrede, R., Hume, C., and Cook, T.: Measurement of ozone and water vapor by Airbus in-service aircraft: The MOZAIC airborne program, an overview, J. Geophys. Res., 103, 25 631-25642, 1998.

Ovarlez, J., Gayet, J.-F., Gierens, K., Ström, J., Ovarlez, H., Auriol, F., Busen, R., and Schumann, U.: Water vapor measurements inside cirrus clouds in northern and southern hemispheres during INCA, Geophys. Res. Lett., 29, doi:10.1029/2001GL014440, 2002.

Spichtinger, P., Gierens, K., and Read, W.: The statistical distribution law of relative humidity in the global tropopause region, Meteorol. Z., 11, 83-88, 2002.

Spichtinger, P., Gierens, K., and Read, W.: The global distribution of ice-supersaturated regions as seen by the microwave limb sounder, Q. J. Roy. Meteorol. Soc., 129, 3391-3410, 2003. 